\title{
Peribulbar block for corneal transplantation: Efficacy and Safety.
}

\author{
Xavier J., Gonçalves G., Sá J., Cavaleiro C., Ferreira L., Machado H. \\ Centro Hospitalar do Porto, Department of Anaesthesiology and Intensive Care, Porto, Portugal
}

\section{BACKGROUND}

Corneal transplantation (CT) requires complete akinesia of the extra-ocular muscles and deep anaesthesia of the surgical site. In our institution, CT has usually been performed under GA, due to fear of the 'open-sky' situation. In the last few years, there has been a growing trend towards using peribulbar block (PBB). We believe that PBB can achieve good surgical conditions with a low complication rate and minimal post-operative analgesic requirement.

\section{GOAL OFTHE STUDY}

Evaluate the efficacy and safety of peribulbar block for CT.

\section{MeTHODS}

We conducted a retrospective audit to evaluate CT under PBB in our institution between June and November 2016. Clinical data collected on each patient included age, sex and American Society of Anesthesiologists physical status. Surgery-specific data included procedure and anaesthesia lengths, characteristics of peribulbar block and analgesic drug administration. Postoperative data included analgesic need in the postanesthesia care unit and within 24 hours of stay and clinical outcome at 15 days.

\section{RESULTS}

76 CT were carried out, 20 under PBB. Of these, I was a penetrating keratoplasty and 19 were lamellar keratoplasties. All patients received an inferotemporal injection and 14 (70\%) received an additional injection, either superomedial or caruncular. I\% ropivacaine was the only local anaesthetic used, with a mean dose of $65 \mathrm{mg}(40-90 \mathrm{mg})$. Most patients received additional systemic analgesia, usually paracetamol Ig (90\%) and/ or fentanyl $0.05 \mathrm{mg}(35 \%)$, mostly to provide perioperative comfort. There was I case of partial block failure, requiring significant systemic analgesia (paracetamol Ig, tramadol $200 \mathrm{mg}$ and fentanyl $0.05 \mathrm{mg}$ ) during the intra-operative period. I patient suffered an episode of bradycardia and hypotension shortly after the block injections, which resolved with the administration of $30 \mathrm{mg}$ ephedrine. No patient required conversion to GA. No serious complications were observed at I5-day follow-up.

\section{CONCLUSIONS}

PBB appears to be a safe and effective technique for CT. Further evidence from prospective and randomised trials is required to compare $\mathrm{PBP}$ with $\mathrm{GA}$.

\begin{tabular}{|c|c|c|c|c|c|c|c|}
\hline & CT & PBB & Drug & Analges & ic ne & ed & Outcome \\
\hline & & & & Perioperat & ive & $24 \mathrm{H}$ & \\
\hline 1 & LK & $\mathrm{IT}+\mathrm{C}$ & 60 & - & - & - & - \\
\hline 2 & LK & $\mathrm{IT}$ & 40 & PCT & $\mathrm{FT}$ & - & - \\
\hline 3 & LK & $\mathrm{IT}$ & 40 & PCT & - & - & - \\
\hline 4 & LK & $\mathrm{IT}+\mathrm{SM}$ & 80 & $P C T+P R X$ & - & - & Edema \\
\hline 5 & LK & $\mathrm{IT}+\mathrm{SM}$ & 90 & $\mathrm{PCT}+\mathrm{PRX}$ & - & - & - \\
\hline 6 & LK & IT & 50 & $\mathrm{FT}$ & - & - & - \\
\hline 7 & LK & $\mathrm{IT}$ & 60 & $\mathrm{FT}$ & - & - & - \\
\hline 8 & LK & $\mathrm{IT}+\mathrm{SM}$ & 60 & - & - & - & - \\
\hline 9 & LK & $\mathrm{IT}+\mathrm{SM}$ & 60 & $\mathrm{PCT}+\mathrm{PRX}$ & - & - & - \\
\hline 10 & LK & $\mathrm{IT}+\mathrm{SM}$ & 80 & PCT+PRX & - & - & - \\
\hline 11 & LK & $\mathrm{IT}+\mathrm{SM}$ & 80 & $\begin{array}{l}\text { PCT+PRX } \\
+ \text { TMD }\end{array}$ & & $\mathrm{FT}$ & - \\
\hline 12 & PK & $\mathrm{IT}+\mathrm{SM}$ & 70 & $\mathrm{FT}$ & - & - & - \\
\hline 13 & LK & $\mathrm{IT}+\mathrm{SM}$ & 70 & $\mathrm{PCT}+\mathrm{PRX}$ & - & - & - \\
\hline 14 & LK & $\mathrm{IT}$ & 60 & PCT & - & - & - \\
\hline 15 & LK & $\mathrm{IT}+\mathrm{SM}$ & 80 & $\mathrm{PCT}+\mathrm{FT}$ & - & - & - \\
\hline 16 & LK & $\mathrm{IT}+\mathrm{SM}$ & 60 & PCT & - & - & - \\
\hline 17 & LK & $\mathrm{IT}+\mathrm{SM}$ & 60 & PCT & - & - & - \\
\hline 18 & LK & $\mathrm{IT}+\mathrm{SM}$ & 70 & PCT & - & - & - \\
\hline 19 & LK & $\mathrm{IT}+\mathrm{SM}$ & 60 & $\mathrm{PCT}$ & - & - & $\mathrm{B}+\mathrm{H}$ \\
\hline 20 & LK & IT & 60 & $\mathrm{PCT}+\mathrm{FT}$ & - & PCT & Bruise \\
\hline
\end{tabular}

PCT - Paracetamol; PRX- Parecoxib; FT- Fentanyl; IT- Inferotemporal; SMSuperomedial; LK- Lamellar keratoplasty; PK- Penetrating keratoplastie. 